\title{
Intratympanic steroids in sudden sensorineural hearing loss: myth or magic?
}

\begin{abstract}
Sudden Sensorineural hearing loss (SSNHL) is a medical emergency inwhich etiopathogenesis and consensus on treatment are still largely debatable. SSNHL has been defined as $30 \mathrm{~dB}$ or more sensorineural hearing loss over at least three contiguous audiometric frequencies occurring within 3days or less. Although several vascular, inflammatory, viral and neoplastic causes exist, no specific etiology can be confirmed; thereforeit is considered idiopathic. Steroids form the mainstay of treatment; however, recent experience with transtympanic steroid injections has been very encouraging. The exact number of injections, dosage, steroid form and window of application is still to be determined conclusively. We report a rare case of idiopathic SSNHL with profound hearing loss treated by 6 shots of transtympanic injections which normalised the hearing. We also review the methods and improvement of transtympanic injections from other studies.
\end{abstract}

Volume 6 Issue 2 - 2017

\author{
Virangna Taneja, Shashidhar Tatavarty, Anish \\ Gupta \\ University Hospital Coventry NHS Trust, UK
}

Correspondence: Virangna Taneja University Hospital Coventry NHS Trust House No 16 Windrush grove Birmingham, UK,Tel+447435629610, Email virangnataneja@ymail.com

Received: November 28, 2016 | Published: March 0I, 2017

Keywords: sudden sensorineural hearing loss, transtympanic steroids

Abbreviations: ISSHL, idiopathic sudden sensorineural hearing loss; SSNHL, sudden sensorineural hearing loss; IT, intratympanic; PET, pressure-equalizing tube

\section{Introduction}

Idiopathic sudden sensorineural hearing loss (ISSHL) is a clinical diagnosis characterised by a sudden deafness of cochlear or retrocochlear origin, in the absence of a clear precipitating cause. Sudden sensorineural hearing loss affects 5 to 20 per 100,000 population, with about 4000 new cases per year in the United States. ${ }^{1,2}$

The diagnosis of idiopathic sudden sensorineural hearing loss remains obscure. Different theories attempt to explain this problem, including disturbance of cochlear blood flow, viral infections, autoimmune disease and Reissner's membrane rupture. ${ }^{3-5}$ Theories presently favoured include a viral or vascular event within the cochlea giving rise to a sudden elevation in hearing thresholds and a degradation in speech discrimination. The natural history is variable, with some patients suffering from permanent hearing threshold changes, whilst others recover some degree of hearing following the insult. $^{4}$

The development of rational treatments for ISSHL has been hampered by uncertainty over the aetiology of the condition; proposedtreatments have been based upon hypotheses of aetiology rather than firm evidence. Treatment modalities trialled include the use of individual or combination agents of vasodilators, diuretics, anticoagulants, plasma expanders, corticosteroids, contrast dye and hyperbaric oxygen. ${ }^{4}$ Evaluation of treatments has been hampered by the low incidence of ISSHL, and the tendency for hearing to recover spontaneously (65\% to $66 \%){ }^{6}$ This has made the contribution of the treatment to hearing recovery difficult to evaluate. Several steroids have been tried for the management via oral, intravenous and transtympanic route. Oral prednisolone, Intravenous 21-amino glucocorticoid steroids, such as methylprednisolone, is commonly prescribed to treat this condition, but their usage is associated with potential side effects. ${ }^{5-9}$ The specific action of steroids in the cochlea is uncertain but their use has been based on their ability to decrease inflammation and oedema. However, there are a wide range of side effects relating to short-term steroid use including glucose intolerance, hypertension, adrenal suppression, gastro-intestinal bleeding and altered mental states. The duration of steroid usage for the treatment of ISSHL is very short (only about two weeks) compared with regimens used to treat chronic disease. The potential side effects from very short, sharp courses of steroids are therefore fewer than those from longer-term use.

A more recent method of corticosteroid delivery is the intratympanic (IT) route.

\section{Case history}

A 66-yr-old male presented with sudden inability to hear in his right ear. There was no history of trauma or any discharge from the ear. The patient complained of associated tinnitus in his right ear.

On examination bilateral tympanic membrane was normal with negative Rinne'stest using 256, 512 and $1024 \mathrm{~Hz}$ tuning fork. Audiometry was suggestive of profound hearing loss in the right ear.

\section{Investigation}

i. MRI Brain (with Contrast) and Ear shows age related changes of cerebral atrophy with chronic ischemic changes; No evidence of Acoustic Neuroma on either side.

ii. Carotid and Vertebral Doppler Artery showed Minor Plaque in both common carotid bulbs.

iii. Non Contrast CT PNS revealed mild Sinusitis; mild deviation of the Nasal Septum and Polyp in Right Maxillary Sinus.

iv. Patient was started on Steroid (Wysolone Tablets) $60 \mathrm{mg}$ from 26th Feb to 9th March 2013, with tapering doses ie. $40 \mathrm{mgs}$ from 10th to 13th March followed by 20mg from 14th till 16th March 2013. Repeat PTA revealed no improvement (Profound SNHL was present).

\section{Treatment}

Patient was then started on Steroid injections (Dexamethasone) $4 \mathrm{mg} / \mathrm{ml}$ dose (9th, 11th and 14th March). We used $0.9 \mathrm{ml}$ steroid with 
$0.1 \mathrm{ml}$ xylocaine to instil intratympanically. Injection was given in the middle of inferior quadrant to prevent injury to underlying labyrinth. Xylocaine was used as a marker; as steroid mixed xylocaine will cause dizziness to patient if inadvertently went into labyrinth. It also provides some surface anaesthesia over the area of promontory. These therapies have considerable side effects. Different types of treatment entail different risks. The clinician should be aware of these potential adverse drug events, including allergic reactions, bleeding, hypotension, arrhythmias, seizures, circulatory collapse, and drug interactions.

\section{Outcome and follow up}

On $18^{\text {th }}$ March PTA showed significant improvement (Moderate Sensorineural Hearing Loss in Right Ear - 51.66dB). As a result of significant improvement, two more injections were given on $18^{\text {th }}$ and $21^{\text {st }}$ March 2013. On 25 $5^{\text {th }}$ March PTA showed further improvement (Mild Sensorineural Hearing Loss in Right Ear - 40dB). Repeat PTA on $8^{\text {th }}$ April showed further improvement (Mild Hearing Loss in Right Ear-35dB). Audiometeric Evaluation done on $18^{\text {th }}$ April showed further improvement (Near normal Hearing in Right Ear - 10dB) Table 1.

The patient had complete recovery of hearing and did not experience any facial puffiness, adrenal suppression, headache, burning of ears, neck hump, hypertension, restlessness or anxiety.

Figure 1-6 are results of audiogram of patient.

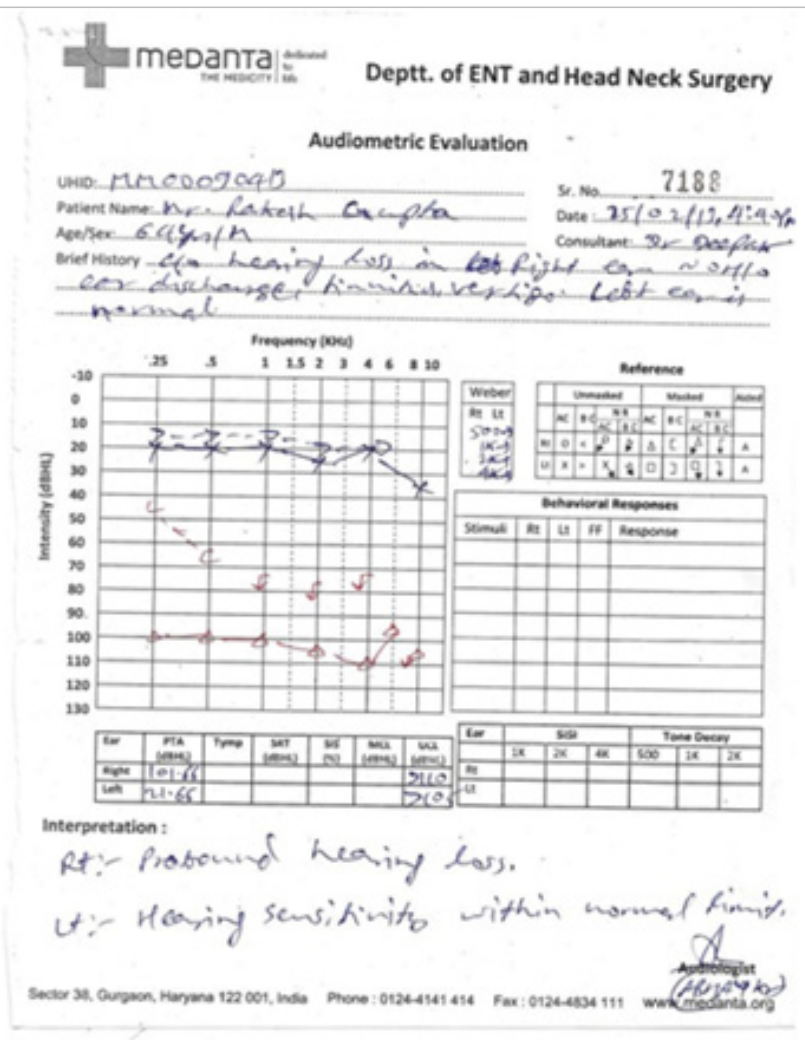

\section{Discussion}

The effectiveness of steroids in the treatment of ISSHL remains unproven. Furthermore, the natural history of ISSHL is highly variable, probably because its pathogenesis is multi factorial. Because spontaneous improvement frequently occurs early after the onset of the hearing loss; the prognosis is worse if the symptoms persist longer. ${ }^{10,11}$ Therefore, prospective randomized controlled trials might be biased and prone to interpretive error, because not all patients can be seen at the same stage of the disease. Further bias could also result from a self-selection process, whereby those who recover quickly do not seek medical care. For maximal treatment outcome, recommended treatment doses of oral prednisone are given at $1 \mathrm{mg} / \mathrm{kg} / \mathrm{d}$ in a single (not divided) dose, with the usual maximum dose of $60 \mathrm{mg}$ daily, and treatment duration of 10 to 14 days. ${ }^{12}$ Data comparing treatment protocols are limited, but one representative regimen uses the maximum dose for 4 days, followed by a 10 -mg taper every 2 days. ${ }^{13}$

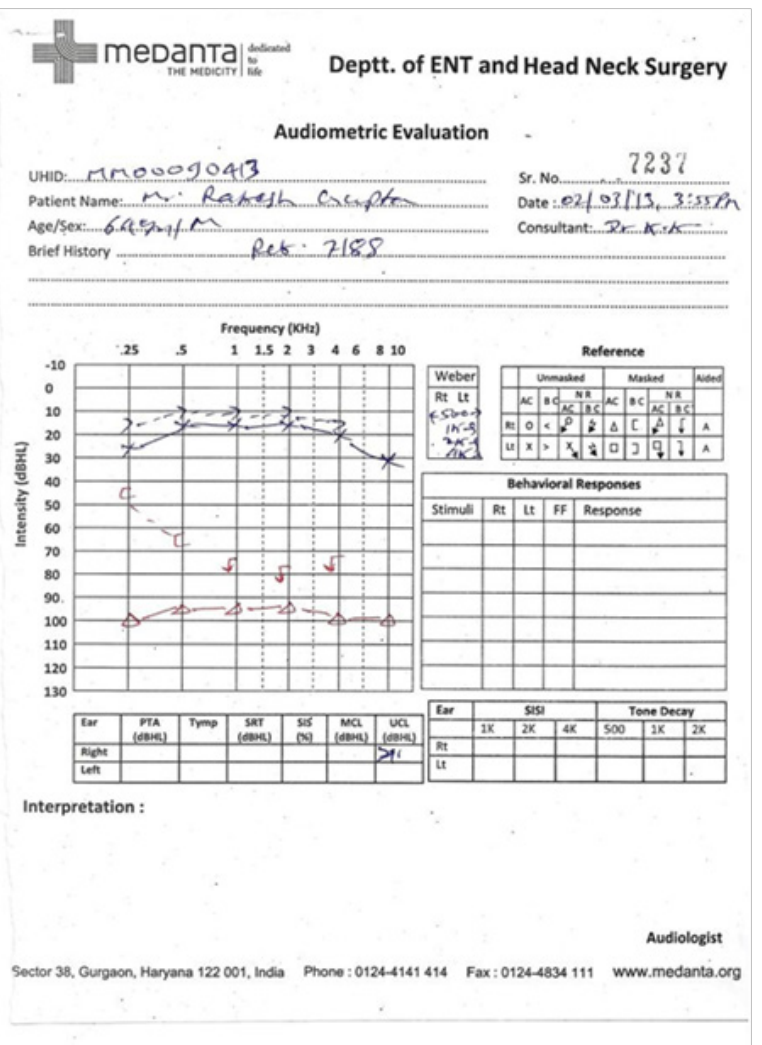

One regimen of initial treatment used combination of oral and intratympanic (IT) steroids for patients with profound hearing loss, in an effort to improve the poor prognosis. They had a positive effect in only 3 of 25 patients. ${ }^{14}$ However, a combination of a high-dose prednisone taper with IT steroids resulted in partial or complete hearing recovery in 14 of 16 patients. ${ }^{15}$ Another study combining oral and IT corticosteroids did not show a difference in hearing recovery compared with corticosteroids alone. ${ }^{16}$ A recent study proposed IT treatment as the sole initial treatment. ${ }^{17}$ Their protocol consisted of early injections for 3 consecutive days, with only 3 of 34 patients failing to improve. A systematic review concluded that IT steroids can be a valuable solution for patients with ISSNHL who either cannot tolerate systemic steroid therapy or are refractory to it. ${ }^{18}$ For patients with diabetes who cannot take systemic corticosteroids, IT steroids may be an alternative. ${ }^{19,20}$

Intratympanic steroids are usually administered as either dexamethasone or solumedrol (methyl prednisolone). ${ }^{21}$ Agents such as histamine and hyaluronic acid have been shown to facilitate transport of the corticosteroid across the round window membrane in laboratory studies. ${ }^{22,23}$ Intratympanic corticosteroids appear to affect both immune suppression and ion homeostasis. ${ }^{24}$ 


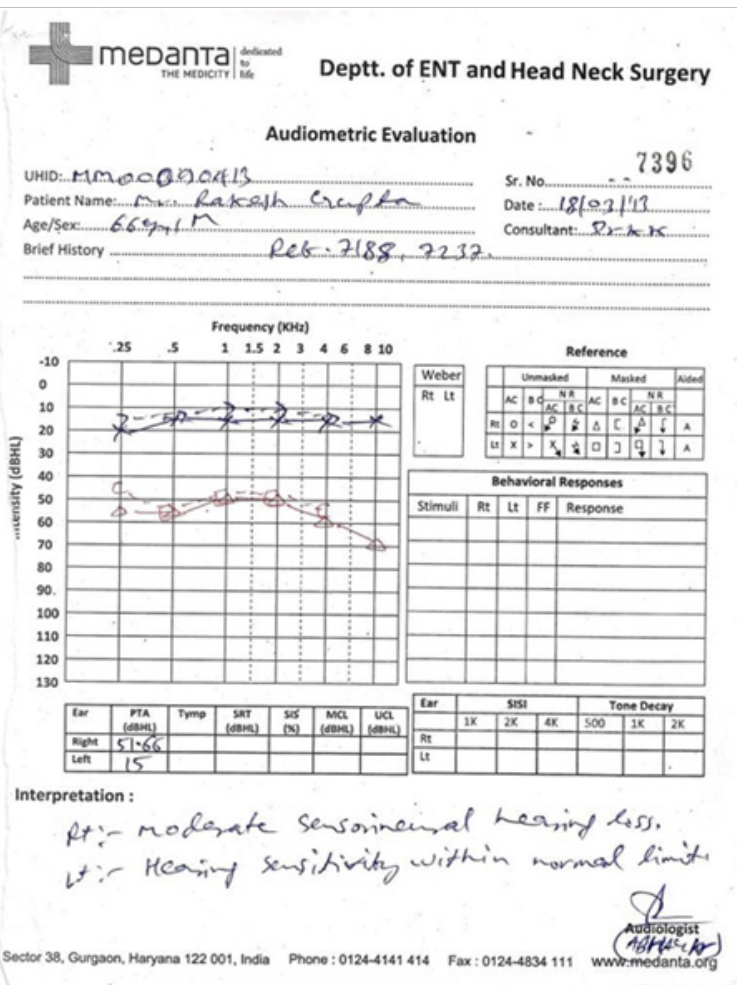

medanTa

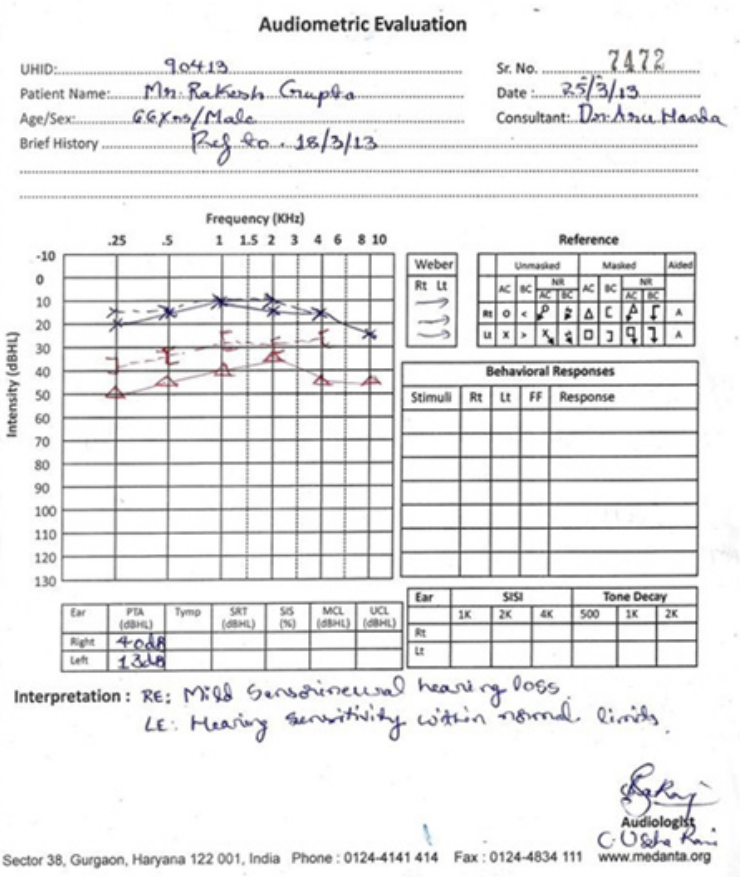

Corticosteroid concentrations vary widely between studies; most studies on IT corticosteroids refer to dexamethasone 10 to $24 \mathrm{mg} / \mathrm{mL}$ and solumedrol $30 \mathrm{mg} / \mathrm{mL}$ and higher. Higher concentrations appear to have better outcomes.
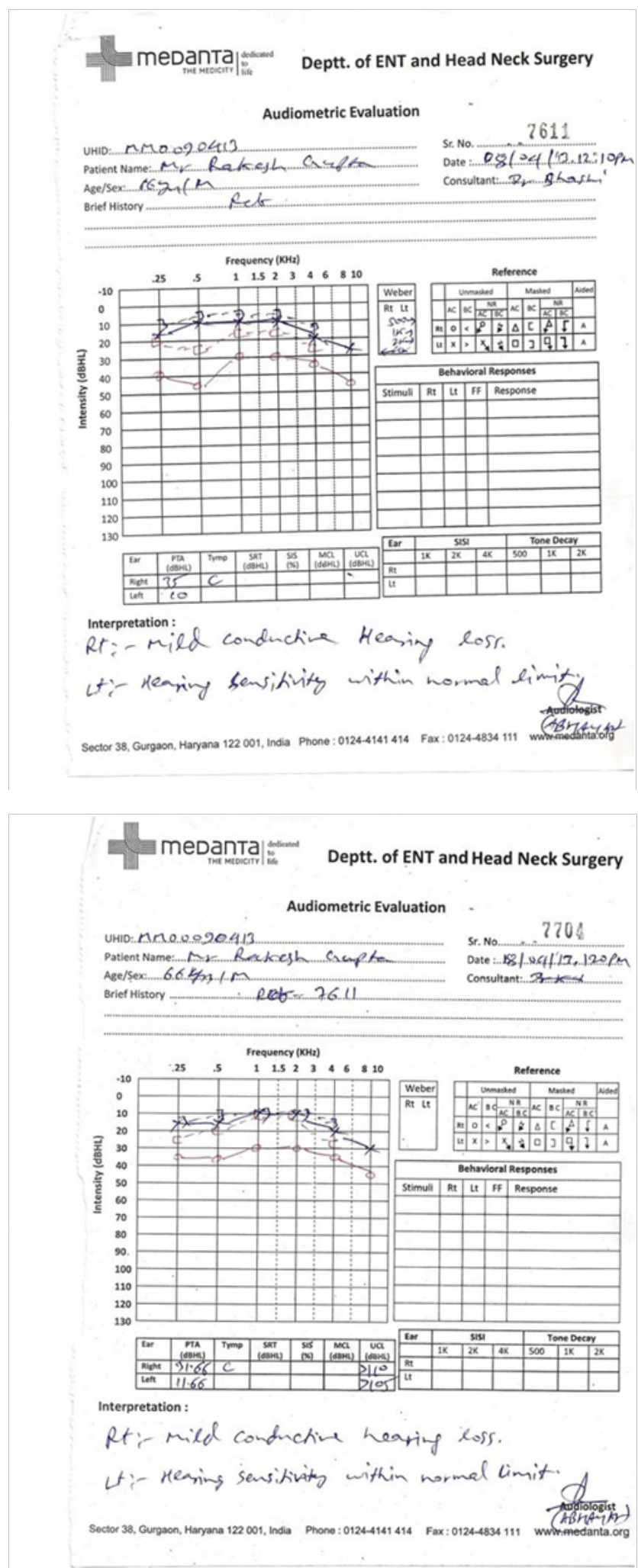

The frequency of IT steroid administration also varies widely between studies, from self-administration by the patient across a pressure-equalizing tube (PET) several times per day to physician administered for several consecutive days to once weekly or less. Moreover, IT corticosteroids have been reported as primary, 
secondary, or salvage treatment. Although with less potential toxicity than systemic corticosteroid treatment, IT corticosteroids can also have adverse effects. These are infrequent but include pain, transient dizziness, infection, persistent tympanic membrane perforation, and possible vasovagal or syncopal episode during injection, cost, and multiple office visits.

The steroids are delivered to the middle ear and then absorbed and diffused through the round window membrane into the inner ear. Intratympanic steroids may be delivered via a needle through the tympanic membrane or may be placed into the middle ear through a tympanostomy tube or a myringotomy (incision in the eardrum). Steroids may also be delivered to the round window via a microcatheter, a MicroWick, ${ }^{25}$ hydrogel applications, and nanoparticles. Transtympanic needle or tympanostomy tubes are the most frequently used. ${ }^{26}$ The IT delivery route has additional benefit of avoiding the considerable side effects of further systemic steroid therapy.

Intratympanic steroids very rarely cause changes in serum glucose levels in patients with diabetes. ${ }^{27}$ They may also be given to patients with cataracts, myasthenia gravis, and glaucoma. ${ }^{28}$ The principal risk appears to be a persistent tympanic membrane perforation at the injection site. This complication, however, is rare and frequently resolves spontaneously or with a paper patch myringoplasty in the office. Existing studies showed considerable variability in the dose and concentration of steroids administered, the timing, frequency, and total number of injections (ranging from one to several to continuous); and drug selection (dexamethasone and methylprednisolone)..$^{18}$ This high degree of variability makes it difficult to compare the results across studies. Despite this variability, 3 of the 4 RCTs evaluating intratympanic steroids as salvage therapy found that IT steroids improved hearing outcomes beyond placebo. Hearing improvement occurred in $53 \%$ to $90 \%$ of patients. ${ }^{29,30}$ Table 2 .

IT steroids are to be started on the 1st day when patient approaches the doctor. It can be used as a main stay of treatment or as an adjuvant treatment with other modalities.

IT steroids have been proven to play an essential role in Meniere's disease and autoimmune inner ear disease apart from SSNHL

Table I Treatment Strategy followed on patient

\begin{tabular}{lll}
\hline 26th February- 9th March & Oral Steroids (4mg/ml Dose) & Profound Hearing Loss \\
9th March & Inj Dexamethasone intratympanically & Profound Hearing Loss \\
IIth March & Inj Dexamethasone intratympanically & \\
14th March & Inj Dexamethasone intratympanically & \\
I8th March & Inj Dexamethasone intratympanically & $\begin{array}{l}\text { Moderate Hearing Loss } \\
\text { 2Ist March }\end{array}$ \\
I8th Dexamethasone intratympanically & Mild Hearing Loss \\
\hline
\end{tabular}

Table 2 Comparison of various modes of instilling IT steroids with their results

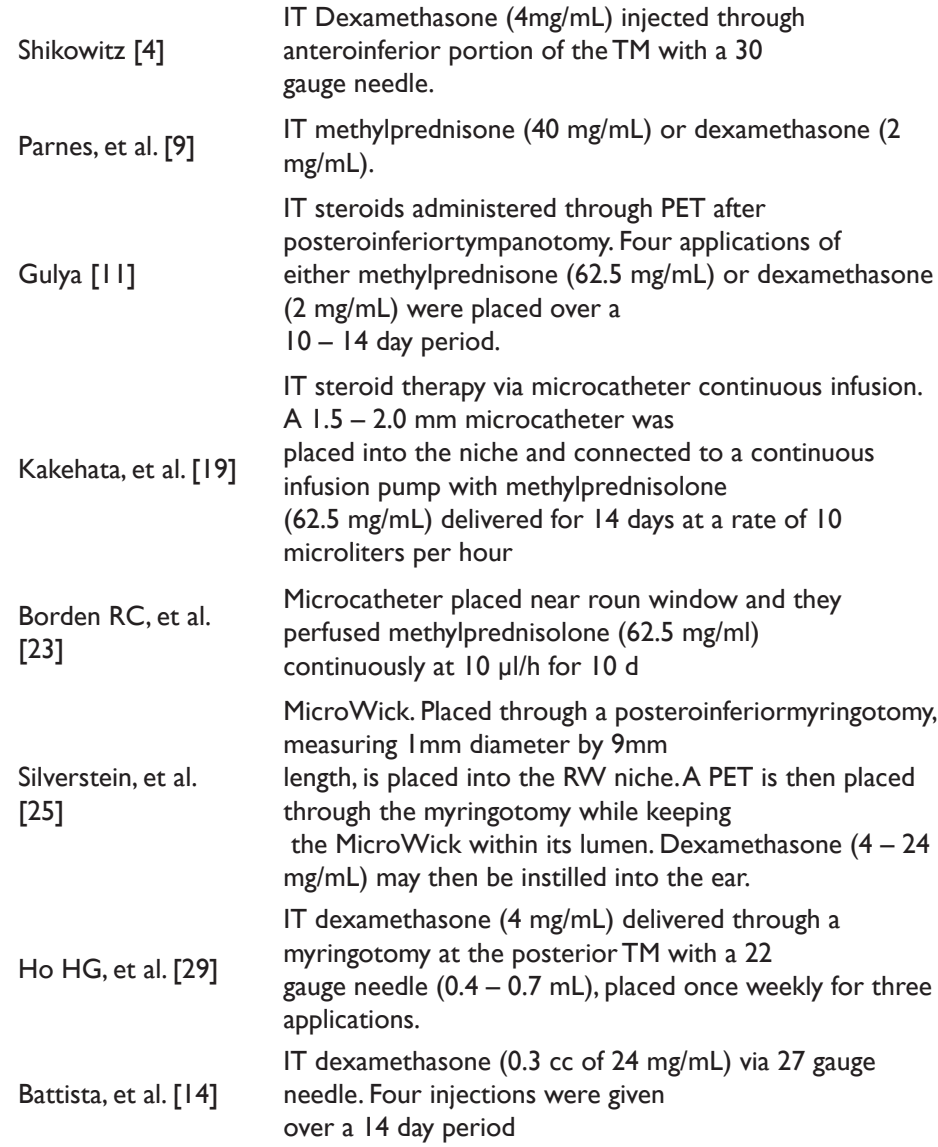

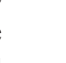


Table Continued...

Xenellis, et al. [30]

IT methylprednisolone $(40 \mathrm{mg} / \mathrm{mL})$ with a 2 I gauge needle, four times over I5 days.

$0.9 \mathrm{ml}$ IT dexamethasone with $0.1 \mathrm{ml}$ Xylocaine instilled in middle of inferior quadrant.

5 doses given over a span of I4days.
47\% treated with IT steroids had improvement in PTA of at least $10 \mathrm{~dB}$.

Max benefit achieved

Patient showed complete recovery

\section{Learning points}

a. Although the effectiveness of steroids in the treatment of ISSHL remains unproven, we recommend more than 3 doses of intratympanic steroid in cases where patients start showing signs of improvement.

b. IT steroids are to be started on the first day when the patient sees the doctor. It can be used as a main stay of treatment or as an adjuvant treatment with other modalities.

c. Effectiveness depends on the dose and concentration of steroids administered, the timing, frequency, and total number of injections (ranging from one to several to continuous); and drug selection (dexamethasone and methylprednisolone).

\section{Acknowledgments}

None.

\section{Conflicts of interest}

Author declares there are no conflicts of interest.

\section{Funding}

None.

\section{References}

1. Byl FM. Seventy-six cases of presumed sudden hearing loss occurring in 1973: prognosis and incidence. Laryngoscope. 1977;87(5 pt 1):817-825.

2. Mattox DE, Simmons FB. Natural history of sudden sensorineural hearing loss. AnnOtol Rhinol Laryngol. 1977;86(4 pt 1):463-480.

3. Cole RR, Jahrsdoerfer RA. Sudden hearing loss: an update. Am J Otol. 1988;9(3):211-215

4. Shikowitz MJ. Sudden sensorineural hearing loss. Med Clin North Am. 1991;75(6):1239-1250.

5. Thurmond M, Amedee RG. Sudden sensorineural hearing loss: etiologies and treatments. J La State Med Soc. 1998;150(5):200-203.

6. Mattox DE, Lyles CA. Idiopathic sudden sensorineural hearing loss. Am J Otol. 1989;10(3):242-247.

7. Haberkamp TJ, Tanyeri HM (1999) Management of idiopathic sudden sensorineural hearing loss. Am J Otol 20(5): 587-592.

8. Stokroos RJ, Albers FWJ. The etiology of idiopathic sudden sensorineural loss. A review of the literature. Acta Otorhinolaryngol Belg. 1996;50(1):69-76

9. Parnes LS, Sun AH, Freeman DJ. Corticosteroid pharmacokinetics in the inner ear fluids: an animal study followed by clinical application. Laryngoscope. 1999;109(7 pt 2):1-17.

10. Eisenman DJ, Alexander A. Effectiveness of treatment for sudden sensorineural hearing loss. Arch Otolaryngol Head Neck Surg. 2000;126(9):1161-1164.

11. Gulya AJ. Sudden sensorineural hearing loss: an otologic emergency Compr Ther. 1996;22(4):217-221
12. Powell-Tuck J, Bown RL, Lennard-Jones JE. A comparison of oral prednisolone given as single or multiple daily doses for active proctocolitis. Scand J Gastroenterol. 1978;13(7):833-837.

13. Rauch SD. Clinical practice: idiopathic sudden sensorineural hearing loss. N Engl J Med. 2008;359(8):833-840.

14. Battista RA. Intratympanic dexamethasone for profound idiopathic sudden sensorineural hearing loss. OtolaryngolHead Neck Surg. 2005;132(6):902-905.

15. Battaglia A, Burchette R, Cueva R. Combination therapy (intratympanic dexamethasone + high-dose prednisone taper) for the treatment of idiopathic sudden sensorineural hearing loss. Otol Neurotol. 2008;29(4):453-460.

16. Ahn $\mathrm{JH}$, Yoo $\mathrm{MH}$, Yoon $\mathrm{TH}$, et al. Can intratympanic dexamethasone added to systemic steroids improve hearing outcome in patients with sudden deafness? Laryngoscope. 2008;118(2):279-282.

17. Filipo R, Covelli E, Balsamo G, et al. Intratympanic prednisolone therapy for sudden sensorineural hearing loss: a new protocol. Acta Otolaryngol. 2010;130(11):1209-1213.

18. Seggas I, Koltsidopoulos P, Bibas A, et al. Intratympanic steroid therapy for sudden hearing loss: a review of the literature. OtolNeurotol. 2011;32(1):29-35.

19. Kakehata S, Sasaki A, Oji K, et al. Comparison of intratympanic and intravenous dexamethasone treatment on sudden sensorineural hearing loss with diabetes. Otol Neurotol. 2006; 27(5):604-608.

20. Han CS, Park JR, Boo SH, et al. Clinical efficacy of initial intratympanic steroid treatment on sudden sensorineural hearing loss with diabetes. Otolaryngol Head Neck Surg. 2009;141(5):572-578.

21. Parnes LS, Sun AH, Freeman DJ. Corticosteroid pharmacokinetics in the inner ear fluids: an animal study followed by clinical application. Laryngoscope. 1999;109(7 pt 2):1- 17

22. Chandrasekhar SS, Rubinstein RY, Kwartler JA, et al. Dexamethasone pharmacokinetics in the inner ear: comparison of route of administration and use of facilitating agents. Otolaryngol Head Neck Surg. 2000;122(4):521-528.

23. Borden RC, Saunders JE, Berryhill WE, et al. Hyaluronic acid hydrogel sustains the delivery of dexamethasone across the round window membrane. Audiol Neurootol. 2011;16(1):1-11.

24. Hamid M, Trune D. Issues, indications, and controversies regarding intratympanic steroid perfusion. Curr Opin Otolaryngol Head Neck Surg. 2008;16(5):434-440.

25. Silverstein H, Jackson LE, Rosenberg SI. Silverstein Microwick ${ }^{\mathrm{TM}}$ for treatment of inner ear disease. Oper Techn Otolaryngol Head Neck Surg. 2011;12(3):144-147.

26. McCall AA, Swan EE, Borenstein JT, et al. Drug delivery for treatment of inner ear disease: current state of knowledge. Ear Hear. 2010;31(2):156-165

27. Kakehata S, Sasaki A, Oji K, et al. Comparison of intratympanic and intravenous dexamethasone treatment on sudden sensorineural hearing loss with diabetes. Otol Neurotol. 27(5): 604-608.

28. Banerjee A, Parnes LS. Intratympanic corticosteroids for sudden idiopathic sensorineural hearing loss. Otol Neurotol.2005;26(5):878-881. 
29. Ho HG, Lin HC, Shu MT, et al. Effectiveness of intratympanic dexamethasone injection in sudden-deafness patients as salvage treatment. Laryngoscope. 2004;114(7):1184-1189.

30. Xenellis J, Papadimitriou N, Nikolopoulos T, et al. Intratympanic steroid treatment in idiopathic sudden sensorineural hearing loss: a control study. Otolaryngol Head Neck Surg. 2006; 134(6):940-945.

31. Bennett MH, Kertesz T, Yeung P, et al. Hyperbaric oxygen for idiopathic sudden sensorineural hearing loss and tinnitus. Cochrane Database Syst Rev. 2012;10:CD004739.

32. Gill AL, Bell CN. Hyperbaric oxygen: its uses, mechanisms of action and outcomes. QJM. 2004;97(7):385-395.
33. Conlin AE, Parnes LS. Treatment of sudden sensorineural hearing loss, II: a Meta-analysis. ArchOtolaryngol Head Neck Surg. 2007;133(6):582-586.

34. Reisser $\mathrm{CH}$, Weidauer H. Ginkgo bilobaextract $\mathrm{EGb} 761$ or pentoxifylline for the treatment of sudden deafness: a randomized, reference-controlled, double-blind study. Acta Otolaryngol. 2001; 121(5): 579-584.

35. Mann W, Beck C, Beck C. Calcium antagonists in the treatment of sudden deafness. Arch Otorhinolaryngol. 1986;243(3):170-173.

36. Aarwal L, Pothier DD. Vasodilators and vasoactive substances for idiopathic sudden sensorineural hearing loss. Cochrane Database Syst Rev. 2009;(4):CD003422. 